\title{
Stable brain networks
}

Neuron 98, 439-452.e5 (2018)

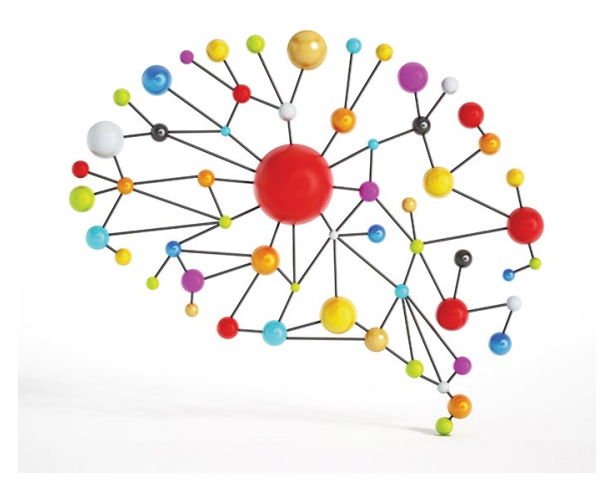

Credit: Cigdem Simsek / Alamy Stock Photo

How different parts of the brain communicate with each other is a central question in neuroscience. So far, the work has led to seemingly contradictory results. Connected areas, so called 'functional networks' have been described at the group level, indicating that humans share the same networks. But differences in network properties have also been identified as markers of psychiatric traits, arguing for dissimilarities between people. Moreover, different tasks have been linked to differences in network activity, suggesting that even within one person, networks can vary over time.

Caterina Gratton, of Washington University, and colleagues report a new study in which they analysed a dataset of repeated measures of the same participants performing the same tasks to tease apart these different potential influences on brain networks. The study shows that network properties are largely shared by all participants in the group, but that there is indeed individual variability. However, measuring the same individual typically yields relatively stable results and the modulation by task is also comparatively small.

The study increases trust in previous conclusions, including links between individual differences in brain networks and traits. The findings also afford specific recommendations for how future network analyses need to be planned and conducted to identify effects at the different levels.

Anne-Marike Schiffer

Published online: 7 June 2018

https://doi.org/10.1038/s41562-018-0365-9 